\title{
Beneficial Effects of Combination Therapy of Sitagliptin and $\beta$-carotene Drugs on Streptozotocin-induced Diabetic Rats
}

\author{
Pulye Roy ${ }^{1}$, Monirul Islam², Mohammad Saiful Islam ${ }^{1}$ and Mamunur Rashid ${ }^{1}$ \\ ${ }^{1}$ Department of Pharmacy, Rajshahi University, Rajshahi-6205, Bangladesh \\ ${ }^{2}$ Department of Pharmacy, Pabna University of Science and Technology, Pabna-6600, Bangladesh
}

(Received: January 8, 2020; Accepted: June 15, 2020; Published (web): July 25, 2020)

\begin{abstract}
Diabetes mellitus (DM), characterized by chronic hyperglycemia is one of the most common chronic diseases affecting millions of people worldwide. A significant component of the risk associated with DM is thought to be because of its abnormal lipid 'triad' profile. All the associated complications are mainly due to imbalance between generation of free radicals and the antioxidant systems like catalase (CAT) and super oxide dismutase (SOD) enzymes. Moreover, increased level of serum glutamate pyruvate transaminase (SGPT) and serum glutamate oxaloacetate transaminase (SGOT) are considered a consequence of hepatocyte damage. The current study was designed to investigate the antihyperglycemic and antidyslipidemic effects, oxidative stress and liver dysfunction indices of fixed dose combination therapy of sitagliptin $[50 \mathrm{mg} / 70 \mathrm{~kg}$ body weight $(\mathrm{BW})]$ and $\beta$-carotene $(5 \mathrm{mg} / 70 \mathrm{~kg}$ $\mathrm{BW}$ ) on streptozotocin (45 mg/kg BW) induced diabetic rats (SIDRs). The study showed that combination therapy induced a significant decrease in blood glucose level (BGL) from 19.09 \pm 0.13 to $6.86 \pm 0.11 \mathrm{mmol} / \mathrm{l}$ in comparison to the control group after daily treatment for three weeks. In case of dyslipidimic effect, combination therapy reduced total cholesterol (TC) (22.96\%), triglycerides (TG) $(17.54 \%)$ and low-density lipoprotein (LDL)-cholesterol $(52.25 \%)$ levels significantly and increased high-density lipoprotein (HDL)-cholesterol level (55.98\%) in comparison with respective diabetic control groups. We also observed that combination therapy lowered SGPT \& SGOT level by $46.39 \%$ and $37.23 \%$, respectively and increased SOD \& CAT enzyme activity by $63.79 \%$ and $48.32 \%$, respectively in comparison with diabetic control groups. These changes were significantly better than those of sitagliptin and $\beta$-carotene monotherapy. The suggestions of our findings of this research are that combination therapy is more potent than their individual monotherapy and may be beneficial for the treatment of diabetic patients with associated complications.
\end{abstract}

Key words: Diabetes, dyslipidemia, oxidative stress, sitagliptin and $\beta$-carotene.

\section{Introduction}

Diabetes is the most common metabolic disorder which is characterized by hyperglycemia, due to insensitivity of insulin and relative or absolute lack of insulin or both (Sattiel et al., 2001; Kumar et al., 2005). Besides high blood glucose level, during diabetes a profound alteration in the concentration and composition of lipid occurs through various metabolic derangements (Sochar, 1995). Insulin deficiency is the major one, which stimulate lipolysis in the adipose tissue and give rise to hyperlipidemia and fatty liver. Furthermore, high glucose promotes intracellular lipid accumulation in vascular smooth muscle cells by impairing cholesterol influx and efflux balance (Xue et al., 2010; Islam et al., 2012). Thus, in diabetes hypercholesterolemia and hypertriglyceridemia often occurs which are independent risk factors that alone or together can accelerate the development of atherosclerosis and progression of atherogenesis, cardiovascular diseases, neuropathy and retinopathy (Hardman et al., 2001; McKenney, 2001; Padmaja, 2010). 
It is reported that generation of free radicals due to oxidative stress are responsible for the disease complications (Garg et al., 1996; Islam et. al., 2019). The body's endogenous antioxidant enzymes e.g. super oxide dismutase (SOD), catalase (CAT), glutathione (GSH) etc. are accountable for the detoxification of free radicals (Jacob, 1995). Antioxidant defense mechanisms of the body are disrupted by the increased productions of free radicals and reactive oxygen species (ROS), cause oxidative damage to the cell membrane and increase lipid peroxidation and these phenomenon ultimately lead to the pathogenesis of many diseases of known and unknown etiology (Sathishsekar and Subramanian 2005). Chronic liver disease e.g. steatohepatitis and nonalcoholic fatty liver disease (NAFLD) are very common in patients having diabetes (Scheen, 2014). Hepatocellular damage occurs due to elevated levels of glutamate pyruvate transaminase (SGPT) and serum glutamate oxaloacetate transaminase (SGOT) enzymes in blood and these two enzymes are the indirect indicators of liver inflammation or injury like NAFLD.

Sitagliptin is a selective inhibitor of dipeptidyl peptidase-4 (DPP-4) enzyme which metabolizes the naturally occurring incretin hormones glucagon-like peptide-1(GLP-1) and glucose-dependent insulinotropic polypeptide (GIP) resulting in enhanced glucose-dependent insulin secretion from the pancreas and decreased hepatic gluconeogenesis (Badyal et. al., 2008). Chronic sitagliptin treatment corrected the glycemic dysmetabolism, hypertriglyceridaemia, inflammation, hypertension, redox status and histopathological lesions of pancreatic tissues, which might be a further advantage in the management of diabetes and its proatherogenic comorbidities (Ferreira et al., 2010). On the other hand, $\beta$-carotene is a naturally occurring and widely distributed carotenoid in foods and it is the precursor of vitamin A. It has potential antioxidant properties due to its chemical structure and interaction with biological membranes and helps to boost up immune system and lower the risk of developing cancer and heart diseases (Riccioni, 2009). Administration of $\beta$-carotene suppressed lipid peroxidation in tissues and improved the glucose tolerance ability of streptozotocin-induced diabetic rats (SIDRs) (Frusho et al., 2002). Therefore, our current investigation was undertaken to evaluate and give a noticeable reference on the possible protective effect of sitagliptin and $\beta$-carotene combination therapy on SIDRs. The present oral hypoglycemic agents and insulin treatment of diabetes is associated with unwanted side effects, limited efficacies and also has limitation in continuation of therapy (Ronald et al., 1991). Therefore, our investigated fixed dose combination therapy containing antidiabetic and antioxidant drugs may be an alternative way of managing long term diabetes with increased risk of dyslipidemia, liver function abnormalities and oxidative stress that exhibits a synergistic effect than their individual monotherapy.

\section{Materials and Methods}

Selection of animal: A total number of 25 Wistar male rats weighing about 250-300 gm (75-90 days old) were acclimatized to the new environmental condition for a period of one week. During the experimental period the rats were kept in a wellventilated animal house at room temperature of $25^{\circ} \mathrm{C}$ and were supplied with standard pellets from ICDDR, B and fresh drinking water. All the rats were kept in cages and maintained with natural 12 hours light and dark cycle. The experiments were performed based on animal ethics guidelines of institutional animal ethics committee principle.

Experimental induction of diabetes: Except the rats of normal control group, all the rats were allowed to fast for 16 hours. Hyperglycemia was induced in each fasted rats by administering streptozotocin (45 $\mathrm{mg} / \mathrm{kg} \mathrm{BW}$ ) in $0.01 \mathrm{M}$ citrate buffer solution ( $\mathrm{pH} 4.5$ ) through intraperitoneal (IP) route. Following the streptozotocin injection, rats were given drinking water supplemented with sucrose $(15 \mathrm{~g} / \mathrm{l})$ for $48 \mathrm{~h}$, to limit early mortality as stores of insulin are released from damaged pancreatic islets. Diabetes was confirmed 3 days later by measuring blood glucose level (BGL) by using glucose test meter (Bioland G423S Test Strip, Germany) using blood sample 
collected from the tail vein of the rats. When the condition of diabetes was established animals with BGL above $11.1 \mathrm{mmol} / \mathrm{l}$ was selected for the study.

Preparation of sitagliptin and $\beta$-carotene: Sitagliptin was used as standard antidiabetic agent and $\beta$-carotene as antioxidative agent in the present study. Each of these two drugs were administered in IP route to hyperglycemic rats $(\mathrm{n}=5)$. Before administration, sitagliptin was dissolved in distilled water and $\beta$-carotene was dissolved in ethanol. Sitagliptin and $\beta$-carotene were administered at a dose of $100 \mathrm{mg} / 70 \mathrm{~kg} \mathrm{BW}$ and $10 \mathrm{mg} / 70 \mathrm{~kg} \mathrm{BW}$, respectively. The combination dose was prepared in solution form at a concentration of sitagliptin (50 $\mathrm{mg} / 70 \mathrm{~kg} \mathrm{BW})+\beta$-carotene $(5 \mathrm{mg} / 70 \mathrm{~kg} \mathrm{BW})$.

Treatment of animals: Rats were randomly assigned into 5 groups containing 5 rats in each group. The repeated dose treatment of animals began on the next day after diabetes induction and this was considered as $1^{\text {st }}$ day of treatment. The doses of sitagliptin, $\beta$-carotene and combination form were administered once in every 24 hours for three weeks in SIDRs as follows:

Group A: Normal control + Distilled water $(0.5 \mathrm{ml})$

Group B: Diabetic control + Distilled water $(0.5 \mathrm{ml})$

Group C: Diabetic + Sitagliptin (100 mg/kg BW)

Group D: Diabetic + $\beta$-carotene $(10 \mathrm{mg} / \mathrm{kg} \mathrm{BW})$

Group E: Diabetic + Sitagliptin $(50 \mathrm{mg} / \mathrm{kg} \mathrm{BW})+\beta-$ carotene $(5 \mathrm{mg} / \mathrm{kg} \mathrm{BW})$

At the end of the treatment period, all the animals were fasted for at least 16 hours and tested for baseline glucose level. After sacrificing the animals, blood samples were collected directly from thoracic artery by heparinized syringe and centrifuged at $4000 \mathrm{rpm}$ for 30 minutes. Serum was separated and then quickly stored at refrigerator for biochemical analysis.

Biochemical analysis: Blood glucose level of serum from each rat was determined by the glucose oxidase method using glucose test meter (Bioland G423S Test Strip, Germany). Serum total cholesterol (TC), Triglycerides (TGs), low-density lipoprotein (LDL)-cholesterol and high-density lipoprotein
(HDL)-cholesterol levels were measured by the UV spectrophotometric method using diagnostic kits (Human, Germany). Serum SGOT and SGPT levels were examined for the estimation of liver functions abnormalities by taking UV absorbance, using diagnostic kits (Human, Germany). Liver was isolated from the sacrificed animals and homogenized in chilled Tris buffer $(10 \% \mathrm{w} / \mathrm{v})$. After then, the homogenate was centrifuged at $4000 \mathrm{rpm}$ for $15 \mathrm{~min}$ in cold centrifuge and the supernatant was analyzed for SOD and CAT activity. The SOD activity was determined by the method of Kakkar $e t$ al. (1984) and CAT activity was assayed by the method of Sinha (1972).

Statistical analysis: The results are expressed as mean \pm SEM using Graph Pad Prism (version 4.0) computer program (Graph pad Software San Diego, CA, USA). We used a one-way analysis of variance (ANOVA), followed by Dunnett's post-hoc test or students paired or unpaired t-test where appropriate. The statistical method applied in each analysis was described in each figure. Results were considered to be significant when $\mathrm{p}$ values were less than 0.05 $(\mathrm{p}<0.05)$.

\section{Results and Discussion}

In this current long term research protocol, effects of monotherapy of drugs (sitagliptin and $\beta$ carotene) and their combination on the different biochemical parameters such as BGL, lipid profile such as TC, TGs, LDL- and HDL-cholesterol, liver functions abnormalities markers like SGOT and SGPT levels, and endogenous antioxidant enzymes like SOD and CAT activity were investigated in SIDRs.

Effects on blood glucose level: The actions of repeated dose treatment for 3 weeks of sitagliptin, $\beta$ carotene and their combination on BGL in normal and SIDRs are shown in the Table 1. When streptozotocin was injected intraperitoneally in animal models, it selectively destroyed pancreatic $\beta$ cells and significantly elevated BGL (21.30 \pm 0.46 $\mathrm{mmol} / \mathrm{l})$ with respect to normal control group $(6.38 \pm$ $0.14 \mathrm{mmol} / \mathrm{l})$. Long term daily dose therapy 
remarkably decrease BGL in case of sitagliptin $(11.29 \pm 0.53 \mathrm{mmol} / \mathrm{l})$ and combination therapy $(6.86$ $\pm 0.11 \mathrm{mmol} / \mathrm{l})$ treated SIDRs. Our results suggested that sitagliptin when combined with $\beta$-carotene more effectively reduced the blood glucose level than mono-therapy in long term SIDRs.

Effects on lipid profile: The dyslipidemia, which was produced in diabetic rats causes alteration in TC, TGs, LDL-C and HDL-C levels. We investigated the lipid profile after three weeks treatment with sitagliptin, $\beta$-carotene and combination of both in
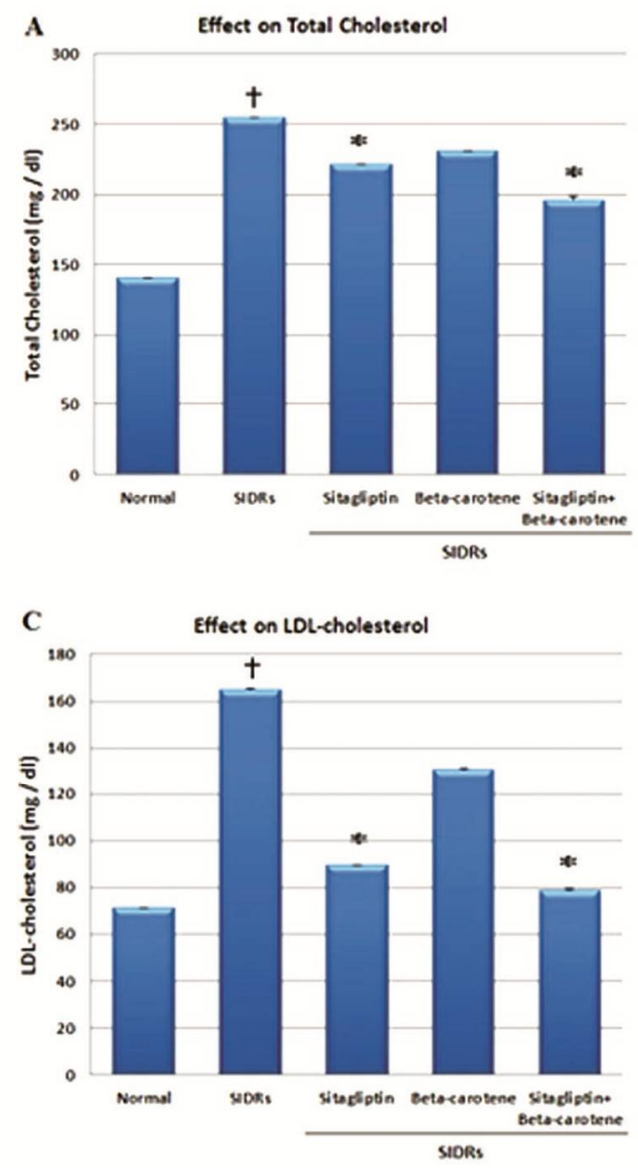

SIDRs. It was found that sitagliptin, $\beta$-carotene and their combination reduced TC level $13.16 \%, 9.36 \%$ and $22.96 \%$ (Figure 1A), TGs level 14.22\%, 11.37\% and $17.54 \%$ (Figure 1B), and LDL-C level $45.79 \%$, $20.73 \%$ and $52.25 \%$ (Figure 1C) and they increased HDL-C level $37.18 \%, 20.77 \%$ and $55.98 \%$ (Figure 1D), respectively when compared with diabetic rats. As the combination therapy showed higher efficacy than that of monotherapy alone, so it might be more effective in lipid profile management than monotherapy alone in long term SIDRs.
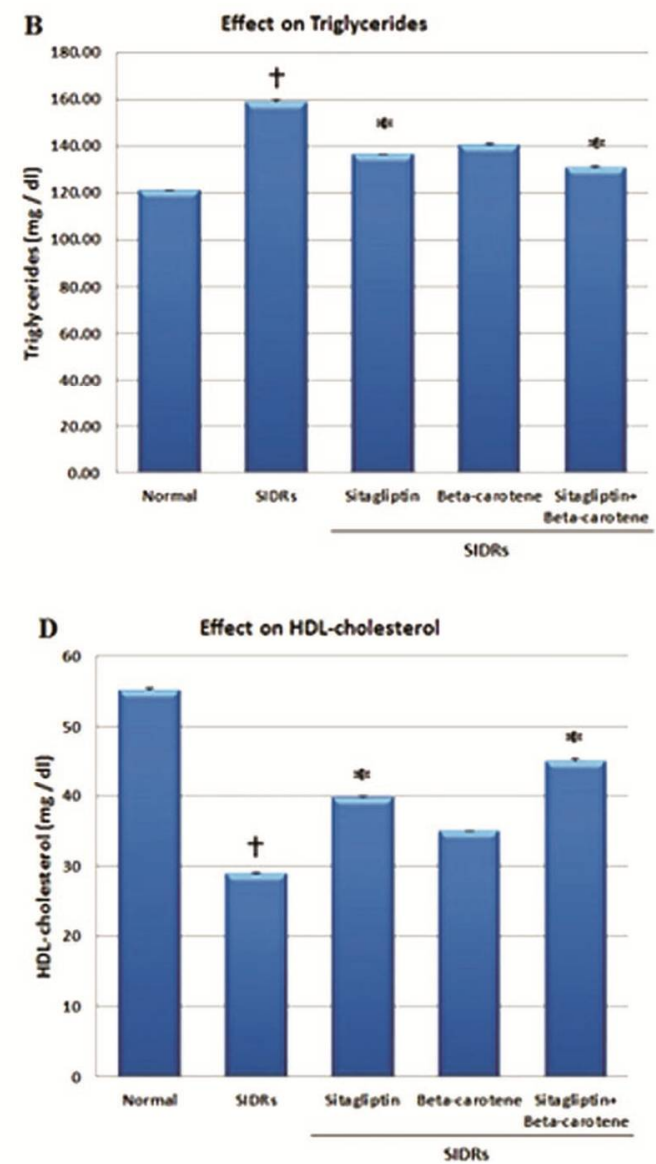

Figure 1. Effects of three weeks repeated dose treatment of sitagliptin, $\beta$-carotene and combination of both on lipid profile: A) TC, B) TG, C) LDL-C and D) HDL-C in SIDRs. Data were presented as mean \pm SEM; $n=5$ in each group. *p $<0.05$ compared to SIDRs and $\dagger(\mathrm{p}<0.05)$ compared to normal group. SIDRs $=$ Streptozotocin-induced diabetic rats.

\section{Effects on liver dysfunction indices}

Effects on SGOT level: At the end of our three weeks treatment protocol with drugs when we tested SGOT level by using serum we found significant rise of SGOT $(\# \mathrm{p}<0.05)$ level in diabetic control group as compared to the normal group. Among the therapies (sitagliptin and $\beta$-carotene alone and their combination dose) sitagliptin alone and combination therapy significantly $(\mathrm{p}<0.05)$ decreased SGOT level as compared to SIDRs in long term daily dose therapy. Combination therapy was more significant than others as depicted in table 2. 
Effects on SGPT level: We also observed raised SGPT value $(\# \mathrm{p}<0.05)$ as like as SGOT level in the SIDRs in comparison to the normal control group. After the long term repeated dose treatment with sitagliptin, $\beta$-carotene and their combination in other diabetic animal groups showed significant $(p<0.05)$ reduction of SGPT level in case of sitagliptin and combination therapy as compared to diabetic control group. However, combination therapy responded more effectively. All these results are outlined in table 2 .

Table 1. Data for the BGL in normal and SIDRs after three weeks treatment.

\begin{tabular}{lcc}
\hline Groups & BGL $(\mathrm{mmol} / \mathrm{l})$ at 0-day & BGL (mmol/l) at 21-Days \\
\hline Normal & $6.38 \pm 0.14$ & $6.83 \pm 0.94$ \\
SIDRs & $21.30 \pm 0.46^{\#}$ & $20.42 \pm 0.16^{\#}$ \\
Diabetic + Sitagliptin & $19.63 \pm 0.20$ & $11.29 \pm 0.53^{*}$ \\
Diabetic + $\beta$-carotene & $20.27 \pm 0.22$ & $17.95 \pm 0.09$ \\
Diabetic + Sitagliptin+ $\beta$-carotene & $19.09 \pm 0.13$ & $6.86 \pm 0.11^{*}$ \\
\hline
\end{tabular}

The data were presented as mean $\pm \mathrm{SEM} ; \mathrm{n}=5$ in each group, $* \mathrm{p}<0.05$ compared to diabetic control group (One way ANOVA followed by Dunnett's test). \#p<0.05 vs. normal group.

Table 2. Data for the SGOT and SGPT levels in normal and SIDRs after three weeks treatment.

\begin{tabular}{lcccc}
\hline \multirow{2}{*}{ Groups } & \multicolumn{2}{c}{ SGOT (units/l) } & \multicolumn{2}{c}{ SGPT (units/l) } \\
\cline { 2 - 5 } & Mean \pm SEM & $(\%)$ Inhibition & Mean \pm SEM & $(\%)$ Inhibition \\
\hline Normal & $29.57 \pm 0.27$ & & $25.14 \pm 0.25$ & \\
SIDRs & $60.27 \pm 1.05^{\#}$ & & $48.70 \pm 0.40^{\#}$ & 40.78 \\
Diabetic + Sitagliptin & $44.44 \pm 0.40^{*}$ & 26.27 & $28.84 \pm 0.40^{*}$ & 23.04 \\
Diabetic + $\beta$-carotene & $52.44 \pm 0.73$ & 13.00 & $37.48 \pm 0.68$ & 46.39 \\
Diabetic + Sitagliptin+ $\beta-$ & $37.83 \pm 0.37^{* *}$ & 37.23 & $26.11 \pm 0.56^{* *}$ & \\
carotene & & & & \\
\hline
\end{tabular}

The data were presented as mean $\pm \mathrm{SEM} ; \mathrm{n}=5$ in each group, ${ }^{*} \mathrm{p}<0.05, * * \mathrm{p}<0.01$ compared to diabetic control group (One way ANOVA followed by Dunnett's test) and \# $<<0.05$ vs. normal group.

\section{Effects on antioxidant enzyme activities}

Effects on SOD activity: The variation of values of SOD enzyme activity at the end of three weeks repeated dose treatment with sitagliptin, $\beta$-carotene and combination of both in normal and SIDRs are depicted in table 3. Animals of diabetic control group had shown significant decreased level of SOD enzyme activity $(\# \mathrm{p}<0.05)$ in comparison with normal control group. We also found that sitagliptin, $\beta$-carotene and combination therapy exclusively increased SOD enzyme activity by 19.46, 49.01 and $63.79 \%$, respectively. From the data, it was cleared that combination therapy expressed most prominent efficacy than that of monotherapy in case of increasing SOD enzyme activity in SIDRs.
Effects on catalase enzyme activity: As of SOD enzyme activity, SIDRs also showed significant decline in CAT activity $(\# p<0.05)$ in comparison with their normal control groups after long term study. The percentage of increment of CAT enzyme activity by the treatment of sitagliptin, $\beta$-carotene and combination in different animal groups as compared to diabetic control group are shown in table 3. Combination therapy expressed most prominent efficacy $(48.32 \%)$ than that of monotherapy in case of increasing CAT enzyme activity in SIDRs.

Diabetes mellitus is a chronic endocrine disorder that is associated with long term damage, dysfunction and failure of various organs especially eyes, kidneys, nerves, heart and blood vessels etc. Simple 
life style adjustments such as a healthy diet and physical activity, often combined with medication have been shown to be effective in promoting a full and healthy life with diabetes. Besides these, combination therapy can be used as an effective way in the treatment of diabetes and diabetes related risk factors especially cardiovascular diseases (CVD). This work was designed to evaluate the possible antidiabetic and hypolipidemic effects, oxidative stress and liver dysfunction indices of combination therapy as well as monotherapy of sitagliptin and $\beta$ carotene in normal and SIDRs. Administration of streptozotocin to rats induces DNA strand breaks in pancreatic $\beta$-cell that stimulates nuclear ADP-ribose synthesis which gives rise to hypoinsulinaemia and hyperglycemia (Howarth et al., 2000). The associated oxidative stress started at early onset of diabetes and increased progressively (Kakkar et al., 1998). This increased oxidative stress may increase glycosylation and oxidation of proteins involved in the pathogenesis of the complications of diabetes. On the other hand, streptozotocin also enhances increased production of free radicals in diabetic rats which are responsible for disruption of insulin action and total body glucose disposal (Brownlee, 2005).

Table 3. Data for the SOD and Catalase enzyme activities in normal and SIDRs after three weeks treatment.

\begin{tabular}{|c|c|c|c|c|}
\hline \multirow[t]{2}{*}{ Groups } & \multicolumn{2}{|c|}{$\begin{array}{c}\text { Super Oxide Dismutase (SOD) } \\
\text { (U/ mg protein) }\end{array}$} & \multicolumn{2}{|c|}{$\begin{array}{c}\text { Catalase (CAT) } \\
(\mu \mathrm{mol} / \mathrm{min} / \mathrm{mg} \text { protein })\end{array}$} \\
\hline & Mean \pm SEM & (\%) Increased & Mean \pm SEM & $(\%)$ Increased \\
\hline Normal & $7.56 \pm 0.24$ & & $85.55 \pm 0.47$ & \\
\hline SIDRs & $4.06 \pm 0.36^{\#}$ & & $53.17 \pm 0.65^{\#}$ & \\
\hline Diabetic + Sitagliptin & $4.85 \pm 0.22^{*}$ & 19.46 & $59.19 \pm 0.69^{*}$ & 11.32 \\
\hline Diabetic $+\beta$-carotene & $6.05 \pm 0.26$ & 49.01 & $70.13 \pm 0.63$ & 31.90 \\
\hline $\begin{array}{l}\text { Diabetic }+ \text { Sitagliptin }+\beta- \\
\text { carotene }\end{array}$ & $6.65 \pm 0.21^{* *}$ & 63.79 & $78.86 \pm 0.44^{* *}$ & 48.32 \\
\hline
\end{tabular}

The data were presented as mean $\pm \mathrm{SEM} ; \mathrm{n}=5$ in each group, $* \mathrm{p}<0.05, * * \mathrm{p}<0.01$ compared to diabetic control group (One way ANOVA followed by Dunnett's test) and \# $<<0.05$ vs. normal group.

After three weeks treatment sitagliptin, $\beta$ carotene and their combination decreased BGL by $44.71 \%, 12.10 \%$ and $66.41 \%$, respectively in comparison to the diabetic rats (Table 1). In this trial, sitagliptin and $\beta$-carotene monotherapy reduced BGL remarkably but not so sharply as like as the combinations therapy in SIDRs. These findings showed similarities with the results of previously published research article (Abbie et al., 2004; Bipin et al., 2013).

Hypercholesterolemia and hypertriglyceridemia had been reported to occur in diabetes mellitus (Riyad, 1988; Tarfa, 1988). In case of diabetes, increased lipid peroxidation occurs where lipids are used much more as fuel to generate ATP. Lipids are made available from the fat depots as LDL and from the liver as VLDL and from the intestine as chylomicrons. This result in a high level of circulating TC, TGs and LDL-C in the blood. Again chronic insulin deficiency causes increased lipolysis in adipose issues and increased entry of free fatty acids (FFA) to the liver, which causes stimulation of triglyceride synthesis (Islam et. al., 2018). This increased synthesis of TGs tends to reduce protein content of lipoproteins, especially in very low-density lipoprotein (VLDL) and LDL while the triglyceride content increases (Winocour et al., 1992). In our current study, diabetic control rats not only showed increased BGL but also showed significant abnormalities in lipid profile (Figure 1). After three weeks treatment, it was found that combination therapy significantly $(\mathrm{p}<0.05)$ reduced TC and LDLcholesterol levels and increased HDL-cholesterol level but moderately decreased TGs level than $\beta$ carotene and sitagliptin monotherapy in SIDRs compared to their diabetic control group (Figure 1). 
The improved lipid profile after treatment with combination dose may be due to reduced lipid peroxide formation. It might be also proposed that the combination therapy may act as inhibitors for enzymes e.g., hydroxyl-methyl-glutaryl-CoA reductase, which participates in de-novo cholesterol biosynthesis. The drugs of the combination may also have synergistic lipid lowering activity.

The enzymes SGPT, a cytosolic enzyme primarily present in the liver and SGOT, a mitochondrial enzyme released from heart, liver, skeletal muscle and kidney are the most sensitive markers employed in the diagnosis of liver diseases. These enzymes leak through the liver cell membrane into the blood circulation while liver cells are injured or died, and serum levels become increase (Zannah et $a l ., 2015)$. It is concluded that diabetes is associated with hepatocellular damage (Thenmohzi and Subramanian, 2009) as diabetic rats show a significant increase of these markers. Long term oral treatment of combination therapy showed significant decrease in serum SGPT and SGOT levels $(\mathrm{p}<0.01)$ than therapy with sitagliptin and $\beta$-carotene alone when compared to their diabetic group. These results suggest that combination therapy offers more protection by preserving the structural integrity of the hepatocellular membrane and our findings are in agreement with the previously published results (Abd El Sattar El Batran et al., 2006).

Hyperglycemia results oxidative stress, which causes free radical formation through various biochemical reactions. Glucose undergoes autooxidation and generates hydroxyl free radicals $(\mathrm{OH})$ (Turko et al., 2001) which again causes autooxidation of unsaturated lipids in plasma and cell membrane. Furthermore, glutathione reserves are depleted through polyol (sorbitol) pathway due to enhanced metabolism of glucose and enhanced production of free radicals (Amira, 2010). These consequences ultimately lead to pathogenesis of many diseases while the endogenous antioxidant enzymes like SOD and CAT are responsible for the detoxification of deleterious oxygen radicals. The activities of SOD and CAT decrease due to inactivation by $\mathrm{H}_{2} \mathrm{O}_{2}$ or by glycation of enzymes. SOD plays a vital role in enzymatic antioxidant defense system by catalyzing the dismutation of superoxide radicals to produce $\mathrm{H}_{2} \mathrm{O}_{2}$ and molecular oxygen, hence diminishing the toxic effects caused by their radical. Again, CAT protects the tissues from highly reactive $\mathrm{OH}$ radicals by catalyzing the reduction of $\mathrm{H}_{2} \mathrm{O}_{2}$ (Lin et al., 2005). In our study, treatment with sitagliptin and $\beta$-carotene increased the activity of these antioxidant enzymes in comparison to their diabetic control rats where $\beta$ carotene alone showed more prominent effect in increment of SOD and CAT activity compared to sitagliptin alone. However, combination therapy increased the SOD and CAT activity most significantly $(\mathrm{p}<0.01)$ than those of monotherapy (Sathishsekar and Subramanian, 2005). It may also reduce the potential glycation of these enzymes or reduce reactive oxygen free radicals and improve the activities of other antioxidant enzymes.

\section{Conclusion}

The beneficial effects of combination therapy of $\beta$-carotene with antidiabetic drug sitagliptin were evaluated by measuring their effects on BGL, serum TC, TGs, LDL-cholesterol, HDL-cholesterol levels, liver functions abnormalities markers like SGOT and SGPT levels, and endogenous antioxidant enzymes like SOD and CAT activity in SIDRs through a three weeks treatment protocol. Results obtained from this study clearly indicated that when sitagliptin was administered in combination with $\beta$-carotene it showed synergistic and promising hypoglycemic properties and reduced dose level of oral hypoglycemic agents, while giving better glycemic control. It also maintained normal lipid profiles, which may represent a protective mechanism against the development of atherosclerosis and has the ability to give hepatic protection and handle oxidative stress, which can reduce the risk parameters associated with diabetes. From our investigation, we can suggest that concurrent administration of $\beta$-carotene with sitagliptin may be more beneficial in the treatment of diabetes and other associated complications like liver 
disorders and increased oxidative stress than individual one, but further study is necessary for the clinical appropriateness of this fixed dose combination therapy.

\section{Acknowledgement}

The authors are thankful to Square Pharmaceuticals Ltd., Bangladesh for its kind gift of sitagliptin and $\beta$-carotene.

\section{References}

Abbie, M.B., Maritim, A.C., Sanders, R.A. and Watkins III, J.B. 2004. Influence of treatment of diabetic rats with combinations of pycnogenol, $\beta$-carotene, and $\alpha$-lipoic acid on parameters of oxidative stress. J. Biochem. Mol. Toxicol. 18, 345-352.

Abd El Sattar El Batran, S., El-Gengaihi, S.E. and El Shabrawy, O.A. 2006. Some toxicological studies of Momordica charantia L. on albino rats in normal and alloxan diabetic rats. J. Ethnopharmol. 108, 236-242.

Amira, A.M. 2010. Oxidative stress and disease. Res. J. Immunol. 3, 129-145.

Badyal, D.K. and Kaur, J. 2008. Sitagliptin: a new class of oral drug for type 2 diabetes. JK Science. 10, 97-98.

Bipin, B.P., Biswaranjan, R., Debasis, G. and Pratap, K.S. 2013. Inhibition of glucose lowering effect of sitagliptin on concurrent use with amlodipine on adrenaline induced hyperglycemic cardiotoxic rat. Asian J. Pharm. Clin. Res. 6, 128-131.

Brownlee, M. 2001. Biochemistry and molecular cell biology of diabetic complications. Nature. 414 (6865), 813-820.

Ferreira, L. and Pinto, F. 2010. Effects of sitagliptin treatment on dysmetabolism, inflammation and oxidative stress in an animal model of type 2 diabetes (ZDF Rat). Mediators Inflamm. 592760, 1-11.

Frusho, T., Kataoka, E., Yasuhara, T., Wada, M. and Innami, S. 2002. Administration of $\beta$-carotene suppresses lipid peroxidation in tissues and improves the glucose tolerance ability of STZ-induced diabetic rats. Int. J. Vitam. Nutr. Res. 72, 71-76.

Garg M.C., Ojha, S. and Bansal, D.D. 1996. Antioxidant status of streptozotocin diabetic rats. Ind. J. Exp. Biol. 34, 264-266.

Hardman, J.G. and Limberd, L.E. 2001. Insulin, oral hypoglycemic agents and the pharmacology of the endocrine pancreas. In: Goodman and Gilman's: The
Pharmacological basis of Therapeutics $(10 \mathrm{ed}$.$) .$ Mcgraw-Hill Company Ltd, USA. pp. 1383-1399.

Howarth, F.C., Qureshi, M.A., Lawrence, P. and Adeghate, E. 2000. Chronic effects of STZ induced diabetes on the ultrastructure of rat ventricular and papillary muscle. Acta Diabetol. 37, 119-124.

Islam, M., Islam, M.S., Zannah, S., Sadik, G. and Rashid, M. 2018. Momordica charantia (Bitter melon) in combination with metformin potentiates hypoglycemic and hypolipidemic effects in alloxan-induced diabetic rats. Bangladesh Pharm. J. 21, 109-117.

Islam, M., Islam, M.S., Zannah, S. and Rashid M. 2019. Combination therapy of Momordica charantia Linn. (Bitter Melon) with antihyperglycemic agent gives increased antioxidative and hepatoprotective actions in alloxan-induced diabetic rats. Bangladesh Pharm. J. 22, 34-40.

Islam, M.S., A. H. M. K. Alam, A.H.M.K., Rahman, M.A.A., Ali, Y., Mamun, A., Rahman, M., Hossain, A.K.M.M., and Rashid, M. 2012. Effects of combination of antidiabetic agent and statin on alloxan-induced diabetes with cardiovascular diseases in rats. J. Sci. Res. 4, 709-720.

Jacob, R.A. 1995. The integrated antioxidant system. Nutr. Res. 15, 755.

Kakkar, P., Das, B. and Viswanathan, P.N. 1984. A modified spectrophotometric assay of superoxide dismutase. Ind. J. Biochem. Biophys. 21, 130-132.

Kakker, R., Mantha, S.V., Radhi, J., Prasad, K. and Karla, J. 1998. Increased oxidative stress in rat liver and pancreas during progression of STZ-induced diabetes. Clin. Sci. 94, 623-632.

Kumar, P.J. and Clark, M. 2005. Diabetes mellitus and other disorders of metabolism. In: Textbook of Clinical Medicine, Saunders (London), pp. 1069-1121.

Lin, Y.F., Tsai, H.L., Lee, Y.C. and Chang, S.J. 2005. Maternal vitamin E supplementation affects the antioxidant capability and oxidative status of hatching chicks. J. Nutr. 135, 2457-2461.

Padmaja, C. and Saramma, G. 2010. M. charantia maintains normal glucose levels and lipid profile and prevents oxidative stress in diabetic rats subjected to chronic sucrose load. J. Med. Food. 13, 520-527.

Riccioni, G. 2009. Carotenoids and cardiovascular disease. Curr. Atheroscler. Rep. 11, 434-439.

Riyad, A., Abdul, G.A.S. and Suleiman, S.M. 1988. Effect of fenugreek and lupine seeds on the development of experimental diabetes in rats. Planta Med. 54, 286290. 
Ronald, K.C. and Shechter, Y. 1991. Insulin, oral hypoglycemic agents and pharmacology of endocrine pancreas. In: The pharmacological basis of therapeutics. Pergamon press, NY, pp. 1463-1495.

Sathishsekar, D. and Subramanian, S. 2005. Antioxidant properties of Momordi cacharantia (bitter gourd) seeds on streptozotocin induced diabetic rats. Asia. Pac. J. Clin. Nutr. 14, 153-158.

Sattiel, A.l. and Khan, C.R. 2001. In insulin signaling and regulation of glucose and lipid metabolism. Nature. 414, 799-806.

Scheen, A.J. 2014. Pharmacokinetic and toxicological considerations for the treatment of diabetes in patients with liver disease. Expert Opin. Drug Metab. Toxicol. 10, 839-857.

Sinha, A.K. 1972. Colorimetric assay of catalase. Anal. Biochem. 47, 389-94.

Sochar, M., Baquer, N.Z. and McLean, P. 1995. Glucose underutilization in diabetes: Comparative studies on the change in activities of enzyme of glucose metabolism in rat kidney and liver. Mol. Physiol. 7, 51-68.

Tarfa, S.P., Joseph, P.K. and Augusti, K.T. 1988. Preliminary studies on the antidiabetic effects of cabbage (Brassica oleracea van capitata L.) oil on streptozotocin-diabetic rats. Curr. Sci. 57, 32-33.
Thenmohzi, A.J. and Subramanian, P. 2009. Antioxidant potential of Momordica charantia in ammonium chloride-induced hyperammonemic rats. Evid. Based Complement. Alternat. Med. 2011, 1-7.

Turko, I.V., Marcondes, S. and Murad, F. 2001. Diabetes associated nitration of tyrosine and inactivation of succinyl-CoA: 3-oxoacid CoA-transferase. Am. J. Physiol. Heart Circ. Physiol. 281, H2289-H2294.

Winocour, P.H., Durrington, P.N., Bhatnagar, D., Ishola, M., Arro, S.I. and Mackness, M. 1992. Abnormalities of VLDL, IDL, and LDL characterize insulin dependent diabetes mellitus. Arteriosc. Thromb.12, 920-928.

Xue, J., Yuan, Z., Wu, Y., Liu, Y., Zhao, Y., Zhang, Y., Tian, Y., Liu, W. and Kishimoto, C. 2010. High glucose promotes intracellular lipid accumulation in vascular smooth muscle cells by impairing cholesterol influx and efflux balance. Cardiovasc. Res. 86, 141150.

Zannah, S., Islam, M., Yusuf, A., Asaduzzaman, M., Shahid, S.M., Al Bari, A.A. and Rashid, M. 2015. Antioxidant in combination with hydroxychloroquine improve antioxidant and hepatoprotective activities in alloxan-induced diabetic rats. Bangladesh Pharm. J. 18, $72-77$. 